\title{
STREPTOCOCCUS PNEUMONIAE CARRIAGE RATE IN HEALTHY PREADOLESCENT DENTAL PATIENTS
}

\author{
Stefano Petti ${ }^{1}$, Aisha A. A. Sofan ${ }^{2}$, Giuseppe A. M essano ${ }^{1}$ \\ ${ }^{1}$ SAPIENZA UNIVERSITY, DEPARTMENT OF PUBLIC HEALTH AND INFECTIOUS DISEASES, ROME, ITALY; \\ ${ }^{2}$ AL THAWRA HOSPITAL, DEPARTMENT OF DENTISTRY AND MAXILLOFACIAL SURGERY, SANA'A, YEMEN
}

Abstract

Introduction. Streptococcus pneumoniae is the major cause of death for pneumonia in the world, responsible 800,000 deaths annually among children and elderly. Most pneumonia caused by pneumococci are healthcare associated, while the remaining are community acquired. Airborne infections are frequent in dental healthcare settings, but data regarding the risk for pneumococci transmission are lacking.

Aim. To estimate $S$. pneumoniae carriage rate among adolescent dental patients, in order to investigate the risk for pneumococci acquisition and spread in dental healthcare settings. Material and methods. 199 children aged 10-12 years attending the paediatric dentistry section of a dental hospital in Rome underwent oropharyngeal swab samples. Pneumococci were presumptively identified with cultural methods (growth on selective media, alpha haemolysis, bile solubility). Routine exposure to passive smoking, use of antibiotics, recent respiratory tract infections (RTIS) were anamnaestically investigated. Unadjusted and adjusted odds ratios (ORs) and individual probability to carry $S$. pneumoniae were assessed with logistic regression analysis.

Results. Overall S. pneumoniae carriage rate was $11.6 \%(95 \%$ confidence interval, 95CI, 7.2-16.0\%). RTIs were significantly associated with carriage (adjusted OR, 3.3; 95CI, 1.3-8.7), exposure to passive smoking (OR, 2.0; 95CI, 0.8-4.9), male gender (OR, 3.2; 95CI, 0.6-17.1) were marginally associated $(0.05<p<0.20)$. According to the regression model, male patients with recent RTI history and routinely exposed to passive smoking yielded $58.2 \%$ probability to carry pneumococci.

Conclusion. S. pneumoniae carriage rate in healthy preadolescent dental patients was moderately high. Patient's profile could be helpful to identify potential carriers and to adopt transmission-based precautions.

Key words: Streptococcus pneumoniae, healthcare-associated infections, dentistry, airborne infection, environment, infection control

\section{Introduction}

Streptococcus pneumoniae is a widespread colonizer of the upper respiratory tract, particularly in children younger than two years. Colonization of the human mucosa of the upper respiratory tract is determined by many factors, such as availability of resources (e.g., nutrients and space), defensive host factors, presence of toxins or harmful substances and type and number of established bacterial populations. For example, Haemophilus influenzae and Staphylococcus aureus, are other colonizers of the upper respiratory tract, which act in antagonism with S. pneumoniae colonization, while pre-existing pneumococci promote adhesion and colonization of other pneumococci strains ${ }^{1}$. S. pneumoniae is transmitted from person to person by respiratory droplets. Environmental factors responsible for transmission are indoor crowding, associated viral infections, lower humidity and air pollution ${ }^{2}$. In the majority of children these microorganisms are not responsible for disease (see, for example 3-5). However, in susceptible individuals, S. pneumoniae is responsible for mild infections, such as otitis media, sinusitis, conjunctivitis and even most serious diseases, such as pneumonia, meningitis, bacterial endocarditis ${ }^{2}$. The most famous example occurred during the deadly pandemic flu of 1918, when several millions of people from all over the world died by influenza-related pneu-

(C) 2013 Faculty of Medicine in Niš. Clinic of Dentistry in Niš. All rights reserved / (C) 2013 Medicinski fakultet Niš. Klinika za stomatologiju Niš. Sva prava zadržana

\footnotetext{
Address for correspondence:

Prof. Dr. Stefano Petti

Department of Public Health and Infectious Diseases

Sapienza University

P.le Aldo Moro 5, 00185 Rome, Italy

Phone/Fax: +3906 49914667

Email: stefano.petti@uniroma1.it
} 
monia. The majority of these deaths are now attributed to pneumococcal super-infection ${ }^{6-7}$. Today, S. pneumoniae is the major cause of death for pneumonia in the world, allegedly responsible for 826,000 deaths annually among children younger than five years, particularly those with important co-morbidities, such as HIV infection or malnutrition, and, only in US, for 401,000 hospitalizations in elderly aged 65 years or older, particularly smokers, alcohol drinkers, subjects with diabetes, chronic lung disease and immunodeficiency ${ }^{8-9}$.

Pneumonia caused by pneumococci are associated with healthcare (HCAP, healthcareassociated pneumonia) or community acquired (CAP, community acquired pneumonia). HCAP definition is pneumonia developed in patients previously hospitalized in acute care hospitals for two or more days within ninety days of the infection, resided in a nursing home or longterm care facility, received recent intravenous antibiotic therapy, chemotherapy, or wound care within the past thirty days of the current infection, or attended a hospital or haemodialysis clinic ${ }^{10}$. S. pneumoniae is the most frequent etiological agent of HCAPs, being responsible for more than one fourth of all cases, while, for example, $\mathrm{H}$. influenzae and $\mathrm{S}$. aureus are responsible for one tenth and one fortieth, respectively. Thus, S. pneumoniae shares the same ecological niche, the same pattern of transmission and similar risk factors, antibiotic resistance, case-fatality rate as methicillin-resistant S. aureus (MRSA) ${ }^{11}$.

Since infections caused by pneumococci are airborne, it would not be surprising that their transmission occurs during dental therapy. For example, oral fluid retraction, which occurs in $>70 \%$ cases when the dental turbine stops rotating, is responsible for aspiration of oral streptococci, non-pathogenic colonizers of the upper respiratory tract, in dental unit waterlines and subsequent spread in the environment during successive dental therapy ${ }^{12}$. The same pattern of transmission could be thought for pneumococci. However, the risk of infection in dental healthcare settings cannot be based on mere conjectures, but must be founded on the solid basis of scientific evidence ${ }^{13}$. Indeed, while data regarding M RSA in dental healthcare settings are few, but sufficient to assess the risk for infection $^{14}$, persistence in the environment ${ }^{15}$ and effect of different control measures ${ }^{16}$, data regarding S. pneumoniae in dental healthcare settings are lacking and do not allow to assess whether these microorganism pose any risk for infection or whether preventive guidelines are necessary. In absence of direct evidence of ascertained cases of infection transmission, indirect evidence could be drawn by observational studies $^{14}$.

\section{Aim}

The aim of this study was to investigate $S$. pneumoniae carriage rate among dental patients who could, therefore, be responsible for the contamination of the environment during dental therapy, thus promoting acquisition and spread of pneumococci at community level.

\section{M aterial and M ethods}

A consecutive sample was selected from children aged 10-12 years attending the paediatric dentistry section of a dental hospital in Rome (Italy). The minimum sample size was estimated assuming S. pneumoniae carriage rate of $8.5 \%$, based on a previous survey among healthy Italian children aged six years ${ }^{17}$. With the highest acceptable margin of error of 5\% and confidence interval of $95 \%$, the sample size was set at 119 subjects. The study protocol was approved by the ethic committee of the dental hospital and written informed consent to nasal swabbing and anamnestic data collection was obtained from children's parents or guardians.

The morning of the appointment and before the dental treatment, participating children underwent an oropharyngeal sample which was collected by one of the authors expert in microbiological procedures and trained for this purpose. The swab was inserted through the mouth and placed on the posterior wall of pharynx and on tonsils for at least five seconds, paying attention not to touch uvula and tongue. Children must not assume antibiotics or other antimicrobials during one week before the sampling occasion and must not drink or eat anything after breakfast. A questionnaire was administered to the parent or guardian by one of the authors and was used to obtain demographic and clinical characteristics of the enrolled children. The questions were 1) development of respiratory 
tract infections (RTI- rhinitis, tonsillitis, laryngitis, acute otitis media, sinusitis, acute bronchitis, pneumonia) during the month before the sampling occasion; 2) assumption of antibiotics or other antimicrobial the month before the sampling occasion; 3 ) daily exposure to passive smoking at home.

The swabs were immediately plated on plates containing Columbia Agar supplemented with 5\% sheep blood (Becton Dickinson Italia, Buccinasco, Italy), $5 \mu \mathrm{g} / \mathrm{mL}$ gentamicin and were incubated in $10 \% \mathrm{CO} 2$ atmosphere at $37^{\circ} \mathrm{C}$ for $24 \mathrm{~h}$. Alpha-haemolytic colonies were gram stained and tested for bile solubility adding few drops of $2 \%$ sodium desoxycholate solution (Becton Dickinson Italia) on some typical colonies. Plates were then incubated at $37^{\circ} \mathrm{C}$ for $30 \mathrm{~min}$. Disappearance of colonies leaving areas of alpha-haemolysis was indicative of positive test ${ }^{18}$. Further identification tests were not made, therefore, positive samples were considered presumptively positive. This option decreased the internal validity of this study, as uncertainty could lead to S. pneumoniae carriage rate estimate artificially higher or lower than the true rate. For this reason, in order to increase the internal validity of the study leaving unchanged the external validity, that is, the chance to extend the present prevalence estimate to Italian healthy children aged 10-12 years, the power of the test was increased by increasing the sample size from 119 to 186 subjects, which resulted in a decrease in the margin of error from $5 \%$ to $4 \%{ }^{19}$.

Presumptive S. pneumoniae carriage rate was estimated with $95 \%$ confidence interval logistic regression analysis was then used to assess the adjusted ORs. Collinearity between explanatory variables was investigated using the Spearman's correlation coefficient $\rho$. If values higher than 0.5 were detected, only one of the two variables was used in the regression model. Robustness of results was investigated through pseudo-R2 and likelihood ratio $\chi 2$ test.

Since the aim of this study was to decrease the chance that airborne pneumococci are spread in the environment of the dental healthcare settings during therapy, thus decreasing the diffusion of these microorganisms at community level, using the coefficients estimated by the multiple regression analysis a probabilistic model was built which provided the chance (expressed in percentage) that a child was S. pneumoniae carrier according to the aforementioned anamnestic variables. Only variables which provided p-values lower than 0.2 in the multiple logistic regression model were considered.

\section{Results}

The study was made between September and December 2011. The number of invited children was 223. Parents or guardians of 24 of them did not provide the consent to swabbing $(n=15)$, anamnestic data collection $(n=4)$, or both $(n=5)$. One hundred ninety-nine children remained and were included in the sample (participation rate, $89.2 \%$ ), their mean age was 11.3 years, al most one half of them was aged 12 years. Females were 106 and males 93 (Table 1). Incidence of reported RTIs during the month

Table 1. General characteristics of the 199 sampled children.

\begin{tabular}{ll}
\hline Variable & Percentage (number) \\
\hline Age, 10 years & $21.6 \%(43)$ \\
Age, 11 years & $31.2 \%(62)$ \\
Age, 12 years & $47.2 \%(94)$ \\
Gender, female & $53.3 \%(106)$ \\
Gender, male & $46.7 \%(93)$ \\
Respiratory tract infections during the last month & $27.1 \%(54)$ \\
Antibiotics during the last month & $8.5 \%(17)$ \\
Exposure to passive smoking & $35.2 \%(70)$ \\
\hline
\end{tabular}

(95CI). The association of S. pneumoniae carriage with age class, gender, RTI, routine exposure to passive smoking and recent antibiotic therapy was initially assessed with logistic regression analysis, which provided the odds ratios (ORs) unadjusted for covariates. Multiple before the sampling occasion was $27 \%$, while $8.5 \%$ assumed antibiotics in the same period. Routine exposure to passive smoking was high, namely, 35\%.

S. pneumoniae carriage rate was $11.6 \%$ (Table 2), with minimal differences between the 
three age classes, while carriage rate was higher in males than in females (12.9\% vs. $10.4 \%)$.

Table 2. Presumptive S. pneumoniae carriage rate. Variable Carriage rate (95CI)

$\begin{array}{ll}\text { Age, } 10 \text { years } & 11.63(2.03-21.17) \\ \text { Age, } 11 \text { years } & 11.29(3.41-19.17) \\ \text { Age, 12 years } & 11.70(5.20-18.20) \\ \text { Gender, female } & 10.38(4.57-16.19) \\ \text { Gender, male } & 12.90(6.09-19.71) \\ \text { Total } & 11.56(7.15-16.05)\end{array}$

The unadjusted ORs for presumptive pneumococci carriage were non-significant excluding for subjects with recent RTIs (OR, 2.8; 95CI, 1.2-6.9) (Table 3). Exposure to routine passive smoking resulted in a marginally significant association with carriage (unadjusted
Probability of carrying S. pneumoniae was the highest for males routinely exposed to passive smoking and with recent RTIs (58\%) (Table 4) and was also high among males with recent RTIs (41\%) and females routinely exposed to passive smoking and with recent RTIs $(30 \%)$. Conversely, in females with no episodes of RTIs and not exposed to passive smoking the chance to carry pneumococci was minimal $(6 \%)$.

\section{Discussion}

The present study is one of the papers presented at the workshop "Advances in Infection Epidemiology and Control in Dental Healthcare

Table 3. Unadjusted and adjusted odds ratios (ORs) of the effects of age, gender, incidence of RTIs during the last month, use of antibiotics during the last month and routine exposure to passive smoking on carriage of presumptive S. pneumoniae.

\begin{tabular}{lll}
\hline Variable & Anadjusted OR $(95 \mathrm{CI})$ & Adjusted OR $(95 \mathrm{CI})$ \\
\hline Age, 11 years & $0.97(0.29-3.28)$ & $0.69(0.19-2.59)$ \\
Age, 12 years & $1.01(0.33-3.10)$ & $0.40(0.06-2.79)$ \\
Gender, male & $1.28(0.54-3.06)$ & $3.22(0.61-17.10)$ \\
\hline
\end{tabular}

Respiratory tract infections during the last month $2.84(1.17-6.89) * 3.34(1.28-8.67) *$

Antibiotics during the last month $0.46(0.06-3.60) \quad 0.53(0.06-4.45)$

Exposure to passive smoking $\quad 1.82(0.76-4.37) \quad 1.98(0.80-4.88)$

* statistically significant at $95 \%$ level

Multiple logistic regression model: Pseudo R2: 0.068. Likelihood ratio $\chi 26 \mathrm{df}$ : 9.70; $p=0.13$

OR, 1.82; 95CI, 0.8-4.4; $\mathrm{p}=0.18$ ). Adjustment for covariates provided an increase in the OR estimates for the most important variables, that is, recent RTIs (OR, 3.3; 95CI, 1.3-8.7), routine exposure to passive smoking $(\mathrm{OR}, 2.0 ; 95 \mathrm{CI}$, 0.8-4.9) and male gender (OR, 3.2; 95CI, 0.617.1). These three variables were associated with pneumococci carriage with $\mathrm{p}<0.20$ and were used in the probability model.
Settings", Department of Public Health and Infectious Diseases, Sapienza University, Rome, Italy on February 9th, 2013 $20-26$.

The estimated S. pneumoniae carriage rate in healthy preadolescents was between $7 \%$ and $16 \%$, suggesting that these microorganisms are likely to be spread in dental healthcare settings. Carriage rate studies in young individuals are not frequent. Oropharyngeal carriage rate among 179 medical students from Turkey

Table 4. Probability of carrying S. pneumoniae according to gender, incidence of RTIs during the last month and routine exposure to passive smoking. Categories were ordered according to decreasing probability.

\begin{tabular}{llll}
\hline Gender & Respiratory tract infections & Passive smoking & Probability (\%) \\
\hline Male & Yes & Yes & $58.2 \%$ \\
Male & Yes & No & $41.3 \%$ \\
Female & Yes & Yes & $30.2 \%$ \\
Male & No & Yes & $29.4 \%$ \\
Female & Yes & No & $17.9 \%$ \\
Male & No & No & $17.4 \%$ \\
\hline
\end{tabular}

Female No $\quad$ Yes $11.4 \%$

Female No No $6.1 \%$ 
was close to $20 \% 27$, but it was $0 \%$ among 79 medical students from A ustria ${ }^{28}$. Studies investigating environmental contamination reported consistent results. Indeed, in a Mexican dental clinic pneumococci were detected in $1 \%$ of samples from the cellular phones of staff and patients ${ }^{29}$, while in US, Israeli and Austrian healthcare settings 30,31 they were not detected. These data collectively suggest that S. pneumoniae carrying dental patients and staff are moderately frequent and that pneumococci are not easily detectable in the environment of healthcare settings. Therefore, S. pneumoniae infections potentially acquired in dental healthcare settings are probably not transmitted by dental healthcare workers.

As previously noted, studies on the risk for S. pneumoniae infection among dental patients and staff are lacking. Therefore, it is not possible to make any convincing speculation regarding the necessity of specific control measures. The present data allowed to design the profile of potential S. pneumoniae carriers among young dental patients. Indeed, male subjects with recent RTI and daily exposure to passive smoking are more likely to be S. pneumoniae carriers. These predisposing characteristics are corroborated by a previous Italian study made on young children ${ }^{17}$. In special dental healthcare settings where immune-compromised patients are generally treated, such as elderly, hospitalized patients, HIV positive subjects, oncologic patients, it is advisable to apply the so called transmission-based precautions ${ }^{32}$. Pneumococcal vaccination is one of these precautions, although polysaccharide pneumococcal vaccines do not seem totally effective in preventing invasive pneumococcal disease and death 33 and the Infectious Disease Society of America does not recommend vaccination in any category of healthcare workers, therefore including dental healthcare workers, but recommends vaccination for adults older than 65 years ${ }^{34}$. Influenza vaccination, useful in preventing HCAP and CAP, including those cases attributable to S. pneumoniae, is probably more adequate in preventing pneumococcal transmission and infection, because it decreases the probability to develop RTIs.

\section{Conclusion}

The present study demonstrated that S. pneumoniae carriage rate in healthy preadolescent dental patients was moderately high and that such rate is higher in subjects with history of recent RTIs. Patient's profile could be helpful to identify potential carriers and to adopt transmission-based precautions, although the risk for infection for dental patients and staff is unknown. It is prudent that control of airborne microorganisms and pneumococcal and influenza vaccinations are made by dental healthcare workers who practice in special care units. 


\section{LITERATURA / REFERENCES}

1. Margolis E, Yates A, Levin BR. The ecology of nasal colonization of Streptococcus pneumoniae, Haemophilus influenzae and Staphylococcus aureus: the role of competition and interactions with host's immune response. BMC Microbiol 2010;10:59.

2. Cartwright K. Pneumococcal disease in western Europe: burden of disease, antibiotic resistance and management. Eur J Pediatr 2002;161(4):188-95.

3. Korona-Glowniak I, Malm A. Characteristics of Streptococcus pneumoniae strains colonizing upper respiratory tract of healthy preschool children in Poland. Sci World J 2012;2012:732901.

4. Sanaei Dashti A, Abdinia B, Karimi A. Nasopharyngeal carrier rate of Streptococcus pneumoniae in children serotype distribution and antimicrobial resistance. Arch Iran Med 2012;15(8):500-3.

5. Tóthpál A, Kardos S, Hajdú E, Nagy K, Linden M, Dobay $\mathrm{O}$. Nasal carriage of Streptococcus pneumoniae among Hungarian children before the wide use of the conjugate vaccine. Acta Microbiol Immunol Hung 2012;59(1):107-18

6. Chien YW, Klugman KP, Morens DM. Bacterial pathogens and death during the 1918 influenza pandemic. N Engl J Med 2009;361(26):2582-3.

7. Klugman KP, Astley CM, Lipsitch M. Time from illness onset to death, 1918 influenza and pneumococcal pneumonia. Emerg Infect Dis 2009;15(2):346-7.

8. O'Brien KL, Wolfson LJ, Watt JP et al. Hib and Pneumococcal Global Burden of Disease Study Team. Burden of disease caused by Streptococcus pneumoniae in children younger than 5 years: global estimates. Lancet 2009;374(9693):893-902

9. Wroe PC, Finkelstein JA, Ray GT et al. Aging population and future burden of pneumococcal pneumonia in the United States. J Infect Dis. 2012 May 15;205(10):1589-92.

10. American Thoracic Society, Infectious Diseases Society of A merica. Guidelines for the management of adults with hospital-acquired, ventilator-associated, and healthcare-associated pneumonia. Am J Respir Crit Care Med 2005;171(4):388-416.

11. Carratalà J, Mykietiuk A, Fernández-Sabé N et al. Health care-associated pneumonia requiring hospital admission: epidemiology, antibiotic therapy, and clinical outcomes. Arch Intern Med 2007;167(13):1393-9.

12. Petti S, Moroni C, Messano GA, Polimeni A. Detection of oral streptococci in dental unit water lines after therapy with air turbine handpiece: biological fluid retraction more frequent than expected. Future Microbiol 2013;8(3):413-21

13. Petti S, Polimeni A. The rationale of guidelines for infection control in dentistry: precautionary principle or acceptable risk? Infect Control Hosp Epidemiol 2010;31(12):1308-10

14. Petti S, Polimeni A. Risk of methicillin-resistant Staphylococcus aureus transmission in the dental healthcare setting: a narrative review. Infect Control Hosp Epidemiol 2011;32(11):1109-15.

15. Petti S, De Giusti M, Moroni C, Polimeni A. Longterm survival curve of methicillin-resistant Staphylococcus aureus on clinical contact surfaces in natural-like conditions. Am J Infect Control 2012;40(10):1010-2.

16. Petti S, Polimeni A, Dancer SJ. Effect of disposable barriers, disinfection, and cleaning on controlling methicillin-resistant Staphylococcus aureus environmental contamination Am J Infect Control 2013. doi:pii: S01966553(12)01270-9.

17. Marchisio P, Esposito S, Schito GC et al. Nasopharyngeal carriage of Streptococcus pneumoniae in healthy children: implications for the use of heptavalent pneumococcal conjugate vaccine. Emerg Infect Dis 2002;8(5):47984.
18. O'Brien KL, Nohynek H, World Health Organization. Pneumococcal Vaccine Trials Carriage Working Group. Report from a WHO Working Group: standard method for detecting upper respiratory carriage of Streptococcus pneumoniae. Pediatr Infect Dis J 2003;22(2):e1-11.

19. Petti S. Why guidelines for early childhood caries prevention could be ineffective amongst children at high risk. J Dent 2010;38(12):946-55.

20. Messano GA, Sofan AAA, Petti S. Quality of air and water in dental healthcare settings during professional toothcleaning. Acta Stomatol Naissi 2013; 29(67): 1230-35; doi: 10.5937/asn1367230M.

21. Messano GA, Masood M, Palermo P, Petti S. Predictors of Legionella occurrence in dental unit waterlines of a highly colonized dental hospital. Acta Stomatol Naissi 2013; 29(67): 1236-41; doi: 10.5937/asn1367236M

22. Messano GA, Masood M, Palermo P, Petti S. Prevalence of reactive tuberculin skin test in dental healthcare workers and students. Acta Stomatol Naissi 2013; 29(67): 1242-48; doi: 10.5937/asn1367242M.

23. Messano GA, De Bono V, Architrave R, Petti S. Environmental and gloves' contamination by staphylococci in dental healthcare settings. Acta Stomatol Naissi 2013; 29(67): 1255-59; doi: 10.5937/asn1367255M.

24. Messano GA. Bacterial and fungal contamination of dental hygienists' hands with and without finger rings. Acta Stomatol Naissi 2013; 29(67): 1260-64; doi: 10.5937/ asn1367260M .

25. Petti S, Messano GA, Polimeni A, Dancer SJ. Effect of cleaning and disinfection on naturally contaminated clinical contact surfaces. Acta Stomatol Naissi 2013; 29(67): 1265-72; doi: 10.5937/asn1367265P.

26. Petti S, Messano GA, Scully C. Antibody level and immunity against Hepatitis B virus infection among general dental practitioners. Acta Stomatol Naissi 2013; 29(67): 1273-78; doi: 10.5937/asn1367273P.

27. Güclü E, Yavuz T, Tokmak A et al. Nasal carriage of pathogenic bacteria in medical students: effects of clinic exposure on prevalence and antibiotic susceptibility. Eur Arch Otorhinolaryngol 2007;264(1):85-8.

28. Gualdoni GA, Lingscheid T, Tobudic S, Burgmann $\mathrm{H}$. L ow nasal carriage of drug-resistant bacteria among medical students in Vienna. GMS Krankenhhyg Interdiszip 2012;7(1):Doc04

29. Munoz Escobedo JJ, Castillo Varela L, Chavez Romero PB, Becema Sanchez A, Moreno Garcia MA. Pathogenic Aerobic Bacteria isolated from cellular telephones of staff and students at the Clinic Multidisciplinary (CLIMUZAC) of the unit academic of dentistry UAZ. Arch Venez Farmacol Terap 2012;31(2):23-31.

30. Goldblatt JG, Krief I, Klonsky T et al. Use of cellular telephones and transmission of pathogens by medical staff in New York and Israel. Infect Control Hosp Epidemiol 2007;28(4):500-3.

31. Jeske HC, Tiefenthaler W, Hohlrieder M, Hinterberger $\mathrm{G}$, Benzer A. Bacterial contamination of anaesthetists' hands by personal mobile phone and fixed phone use in the operating theatre. Anaesthesia 2007;62(9):904-6.

32. Harte JA. Standard and transmission-based precautions: an update for dentistry. J Am Dent Assoc 2010;141(5):572-81

33. Babouee B, Widmer AF, Battegay M. Vaccination against pneumococci and influenza. How good is the evidence? Internist 2011;52(3):265-76.

34. Pickering LK, Baker CJ, Freed GL et al Immunization programs for infants, children, adolescents, and adults: clinical practice guidelines by the Infectious Diseases Society of America. Clin Infect Dis 2009;49(6):817-4. 